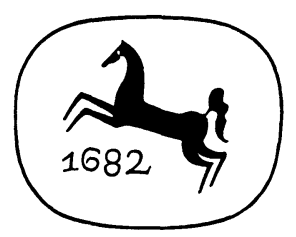

Fachliteratur des Mittelalters 


\title{
Fachliteratur des Mittelalters
}

\author{
Festschrift \\ für Gerhard Eis
}

Herausgegeben

VON

Gundolf Keil, Rainer Rudolf, Wolfram Schmitt und Hans J. Vermeer

J. B. Metzlersche

VERLAGSBUCHHANDLUNG

StUTtgart 
ISBN 978-3-476-99495-0

ISBN 978-3-476-99494-3 (eBook)

DOI 10.1007/978-3-476-99494-3

(C) 1968 Springer-Verlag GmbH Deutschland

Ursprünglich erschienen bei J. B. Metzlersche Verlagsbuchhandlung und Carl Ernst Poeschel Verlag GmbH in Stuttgart 1968. 


\section{Inhaltsverzeichnis}

Vorwort .................. IX

Franz H. BäUmL, Der Übergang mündlicher zur artes-bestimmten Literatur des Mittelalters. Gedanken und Bedenken . . . . . . . . 1

George Fenwick Jones, English Borrowings from Medieval German Fachsprache . . . . . . . . . . . . . . . . .

Eli Sobel, Two Meisterlieder on the Seven Liberal Arts in MS Berlin germ. quart. 414 . . . . . . . . . . . . . . .

James Follan, Manuscripts of Ortolf von Bayerlants 'Arzneibuch'. Their Contents, Exemplifying German Mediaeval „Artesliteratur" . . . . .

Rainer Rudolf, Heinrichs von Langenstein 'Erchantnuzz der sund' und ihre Quellen .. . . . . . . . . . . . . . .

Dieter Richter, Die Allegorie der Pergamentbearbeitung. Beziehungen zwischen handwerklichen Vorgängen und der geistlichen Bildersprache des Mittelalters . . . . . . . . . . . . . . .

Rolf Klemmt, Mitteilung zu einer „Anaphorischen Betrachtung“ der Spätgotik . . . . . . . . . . . . . . . . . .

Rolf Winau, Christian Mentzels wissenschaftliche Interessen im Spiegel seiner Beiträge in den 'Miscellanea Curiosa' . . . . . . . . . . . . 101

Kalevi Tarvainen, Zur Problematik der sprachlichen Untersuchung historischer Chroniken des Spätmittelalters . . . . . . . . . . 115

Gundolf KeIL, Die Grazer frühmittelhochdeutschen Monatsregeln und ihre Quelle . . . . . . . . . . . . . . . 131

Judica I. H. Mendels, Einiges über die deutsche Hüttensprache im Mittelalter . . . . . . . . . . . . . . . . . . 
Eero Alanne, Das Vordringen der römischen bzw. romanischen Weinbauterminologie in die Nord- und Ostseegebiete . . . . . . . . . . 167

Willy L. Braekman, 'Vander Hulpen des Ghebrecs des Wiins'. Een onbekend Middelnederlands wijntraktaat uit de vijftiende eeuw . . .

Willem F. Daems, Die Clareit- und Ypocrasrezepte in Thomas van der Noots 'Notabel Boecxken van Cokeryen' (um 1510) . . . . . . . . 205

Hans Wiswe, Das Pflanz- und Pfropfbüchlein des Johann Balhorn . . . . 225

Kurt Lindner, Alte deutsche Weidsprüche . . . . . . . . . . . . 245

Gunnar Tilander, Vieux français tres maint . . . . . . . . . 259

Gerhart Hoffmeister, Fischer- und Tauchertexte vom Bodensee . . 261

Wilhelm Rieck, Zur Pathologie der Pferdeseuchen im Mittelalter . . . 277

Heinz Harms, Die pflanzlichen Arzneistoffe einer mittelniederdeutschen hippiatrischen Rezeptsammlung des 16. Jahrhunderts . . . . . . . 293

Gernot Rath ( $\dagger$ ), Montpellier im Urteil des deutschen Mittelalters . . . 307

Heinrich Schipperges, Handschriftliche Untersuchungen zur Rezeption des Petrus Hispanus in die 'Opera Ysaac' (Lyon 1515) . . . . . . . . 311

Eduard SeIdler, Pariser Medizin im 15. Jahrhundert . . . . . . . 319

Christian Probst, Der Weg des ärztlichen Erkennens bei Heinrich von Mondeville . . . . . . . . . . . . . . 333

Walter Pagel und Marianne Winder, Gnostisches bei Paracelsus und Konrad von Megenberg . . . . . . . . . . . . . . . . 359

Karl-Heinz Weimann, Probleme der medizinischen Fachsprache zur Zeit des Vesal . . . . . . . . . . . . . . . . 373

Johannes Steudel, Thymus ................. 381

Egon Schmitz-Cliever, Die medizinhistorische Deutung des Namens 'Rosstraße' in Aachen . . . . . . . . . . . . . . . . . 385

Hans H. Lauer, Zur Überlieferungsgeschichte der Salep-Wurzel . . . . 395

Hans J. Vermeer, Cardo benedicta das edlist krautt. Handschriftentexte aus Wien, Harburg und Böhmen . . . . . . . . . . . . . . . . . 421

WoLfram SchmitT, Zwei weitere Überlieferungen des Büchleins 'Von den gebrannten Wässern' Gabriel von Lebensteins . . . . . . . . . . . 433 


\section{Inhaltsverzeichnis}

Gustav Korlén, Stockholmer Arzneibuchstudien. Ein Forschungsbericht 449

Walter Lawrence Wardale, Some Notes on the Stockholm MS X 113 and the Göttingen MS hist. nat. 51 . . . . . . . . . . . . . 457

Josef Werlin, Ein Rezeptbuch des Kaisers Maximilian I.? Neun dem deutschen Kaiser zugeschriebene medizinische Texte . . . . . . . . . 469

Hans Schabram, Zur Interpretation von 'Beowulf' 2697 ff. . . . . . . 481

Alphons Augustinus Barb, Die Blutsegen von Fulda und London . . . 485

MaNFRed Stürzbecher, Bemerkungen zu lebensmittelhygienischen Bestimmungen in mittelalterlichen deutschen Rechtsaufzeichnungen . . . 495

Hans J. Vermeer, Verzeichnis der Schriften von Gerhard Eis . . . . . . 499

Namen- und Sachregister (bearbeitet von RAINeR Rudolf) . . . . . . 535 


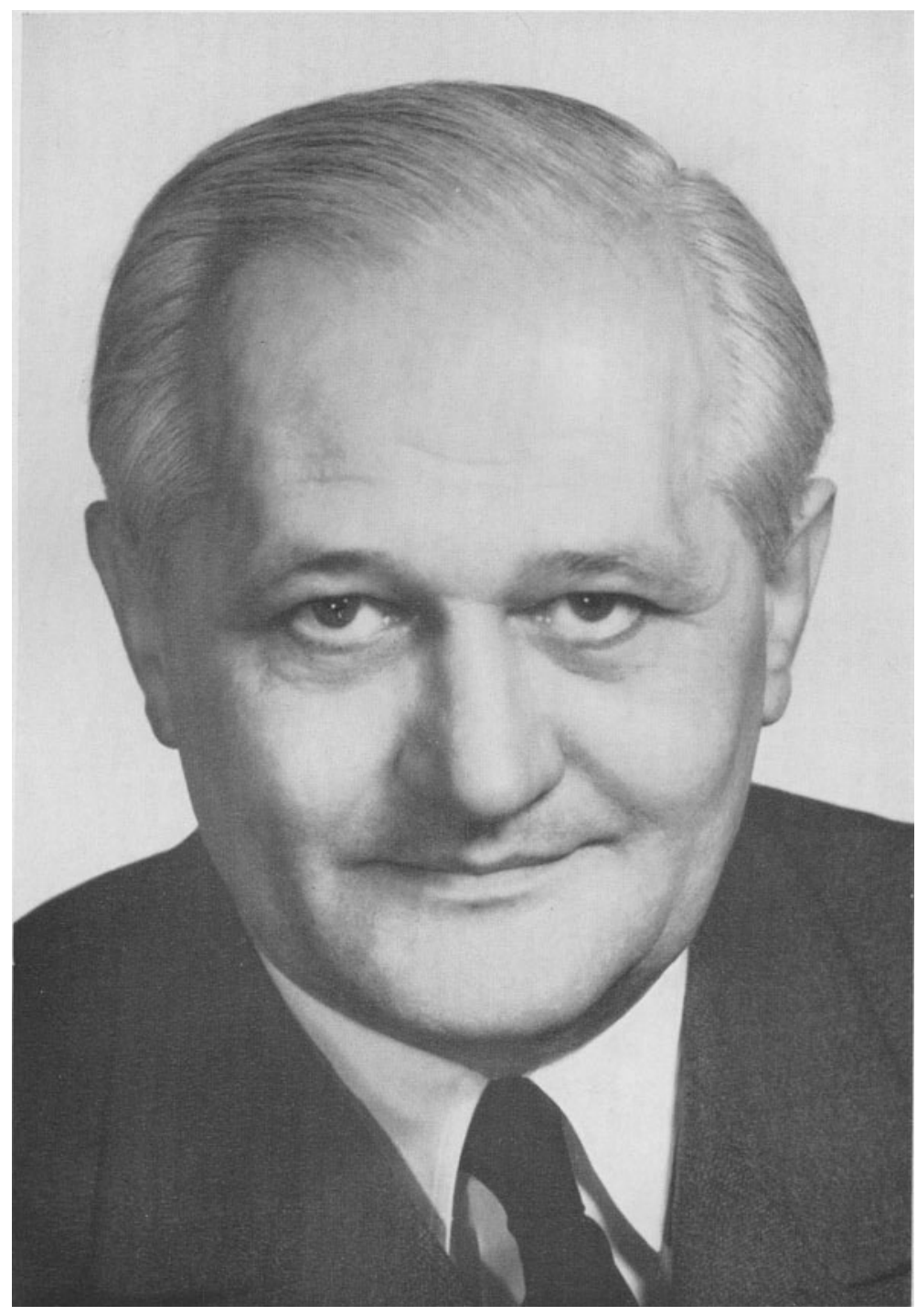

Gerhard Eis 


\section{Vorwort}

Bisher hat sich die Mediävistik in recht unterschiedlichem Ausmaß mit dem reichhaltigen, landessprachlich wie lateinisch überlieferten Quellenmaterial auseinandergesetzt, das als 'Mittelalterliche Fachliteratur' alle in Vers oder Prosa abgefaßten Denkmäler beruflichen und wissenschaftlichen Schrifttums umgreift: von seiten der Kirchen- und Philosophiegeschichte, der politischen und der Rechtsgeschichte hat eine eingehende Beschäftigung mit den geistlichen, philosophischen, historischen und juristischen Texten des Mittelalters zu beachtlichen Ergebnissen geführt, während die eigentlich wissenschaftshistorischen Disziplinen, also die Geschichte der Human- und Veterinärmedizin, der Pharmazie, der Technik und der Naturwissenschaften, in der planmäßigen Auswertung der einschlägigen Literaturkomplexe noch zurückliegen, wenngleich gerade in jüngster Zeit auch hier ein eindrucksvoller Aufschwung zu verzeichnen ist. Auch die philologische Erschließung der mittelalterlichen Geisteswelt krankte lange Zeit - und teilweise noch heute - an einer ungenügenden Berücksichtigung der Fachprosa. Dies gilt insbesondere für die Germanistik, deren Methodik und Resultate auf dem Gebiet der altdeutschen Literatur häufig anfechtbar bleiben, solange sie sich mit der Erfassung der dichterischen Überlieferung begnügen zu können glaubt. Es ist das Verdienst von Gerhard Eis, daß sich hier im Verlauf der letzten drei Jahrzehnte eine Entwicklung angebahnt hat, die auf eine konsequente Erfassung der deutschsprachigen wissenschaftlichen Literatur des Mittelalters ausgeht mit dem Ziel einer auf breiterer Grundlage ruhenden und damit der historischen Wirklichkeit näherrückenden Darstellung der mittelalterlichen deutschen Literatur-, Sprach- und Geistesgeschichte. Dieser junge und zukunftsweisende Zweig der deutschen Philologie ist inzwischen zu ansehnlicher Blüte gelangt; die lebhafte Zustimmung in in- und ausländischen Germanistenkreisen wie auch die Aufgeschlossenheit der Wissenschaftshistoriker, die die Zusammenarbeit mit den Philologen gerade auf dem 
schwierigen Feld der Mittelalterforschung schätzen lernten, haben ein vielschichtiges wissenschaftliches Arbeiten wachgerufen, das die Bedeutung der neuen Forschungsrichtung sinnfällig unterstreicht. Der größte Teil der Arbeit ist freilich noch zu leisten, und auf zahlreichen Gebieten der Fachliteratur steht die Forschung erst am Anfang. Das ausgesprochen vielgestaltige und umfangreiche Schrifttum ist noch keineswegs vollständig ausgewertet, vielfach fehlt es an Textausgaben, und auf weiten Strecken ist die Überlieferung noch nicht einmal gesichtet. Indessen hat sich beim Ordnen der Stoffmassen das Gerüst der mittelalterlichen Artesreihen - der Freien Künste, der Eigenkünste und der Verbotenen Künste -, wie es von der scholastischen Wissenschaftslehre entwickelt wurde, als Arbeitsgrundlage bewährt und den Weg gewiesen zur Erfassung auch derjenigen Fachprosabestände, die außerhalb des geistlichen, historischen und juristischen Bereichs liegen und den Komplex der Artesliteratur ausmachen. Daß sich bei der wissenschaftlichen Bemühung um diese literarischen Stoffe eine enge Verbindung und ein fruchtbares Gespräch der Germanistik mit den Nachbardisziplinen, insbesondere den wissenschaftsgeschichtlichen Fächern, aus der Sache selbst ergibt, kann man in einer Zeit zunehmender Spezialisierung der Wissenschaften als hohen Gewinn verbuchen. Nicht minder bedeutsam erscheint die gerade im Umkreis der Fachprosaforschung so rege und freundschaftliche Zusammenarbeit deutscher und ausländischer Gelehrter, wie sie als kennzeichnendes Merkmal zeitgemäßen wissenschaftlichen Stils gelten kann. Beides, die Überwindung der Grenzen zwischen den Fächern und zwischen den Staaten, spiegelt sich wider in den Beiträgen, die in dieser Festschrift vereint sind. Sie geben darüber hinaus in der Vielfalt ihrer Fragestellungen und Ergebnisse Zeugnis von der Lebendigkeit und Ergiebigkeit des Forschens auf dem Felde mittelalterlicher Fachliteratur. In diesem Sinne seien sie Gerhard Eis, dessen grundlegenden und richtungweisenden Untersuchungen sie mannigfach verpflichtet sind, in Dankbarkeit und Verehrung gewidmet.

Die Herausgeber betrauern den Tod von Gernot Rath, der am 28. September 1967 aus weitgespanntem wissenschaftlichem Wirken herausgerissen wurde. Von den zahlreichen Forschungsvorhaben, die er in letzter Zeit verfolgte, zeugt neben anderem eine kurze Montpellier-Studie, die er unmittelbar vor seinem Tod niederschrieb.

Die Herausgeber 